\title{
Inhaler Technique Education and Exacerbation Risk in Older Adults with Asthma or Chronic Obstructive Pulmonary Disease: A Meta-Analysis
}

\author{
Tiago Maricoto, MD, ${ }^{* \dagger}$ (1) Luís Monteiro, MD, ${ }^{\dagger+}$ Jorge M.R. Gama, PhD, ${ }^{\S}$ \\ Jaime Correia-de-Sousa, $M D,{ }^{I / \|}$ and Luís Taborda-Barata, $M D^{* *+1 * *}$
}

OBJECTIVES: To evaluate the effect of inhaler education programs on clinical outcomes and exacerbation rates in older adults with asthma or chronic obstructive pulmonary disease (COPD).

DESIGN: Systematic review and meta-analysis.

SETTING AND PARTICIPANTS: Older adults with asthma or COPD, either in primary or secondary health care and pharmacy setting.

MEASUREMENTS: We searched the Medline, Embase, and Central databases according to the main eligibility criteria for inclusion: systematic reviews, meta-analysis, clinical trials and quasi-experimental studies; participants aged 65 and older; education on inhaler technique and reporting of disease control and exacerbation rates. We used the Grading of Recommendations, Assessment, Development and Evaluations scale for quality assessment and used a random-effect model with Mantel-Haenszel adjustment to perform a meta-analysis.

RESULTS: We included 8 studies $(4$ randomized, 4 quasiexperimental) with a total of 1,812 participants. The most frequent type of intervention was physical demonstration of inhaler technique, training with placebo devices. Five studies showed significant reduction in exacerbation rates (pooled risk ratio $=0.71,95 \%$ confidence interval $=0.59-0.86 ; \mathrm{p}<.001)$,

From the *Aveiro-Aradas Family Health Unit Aveiro Healthcare Centre, Aveiro, Portugal; ${ }^{\dagger}$ Faculty of Health Sciences, University of Beira Interior, Covilhã, Portugal; "Esgueira + Family Health Unit Aveiro Healthcare Centre, Aveiro, Portugal; ${ }^{\S}$ Centre of Mathematics and Applications, Faculty of Sciences, University of Beira Interior, Covilhã, Portugal; "Life and Health Sciences Research Institute/3B's-PT Government Associate Laboratory, University of Minho, Braga, Portugal; "Horizonte Family Health Unit, Unidade Local de Saúde, Matosinhos, Portugal; **CICS-Health Sciences Research Centre, University of Beira Interior, Covilhã, Portugal;

${ }^{\dagger}$ NuESA-Environment and Health Study Group, Faculty of Health Sciences, University of Beira Interior, Covilhã, Portugal; and the

* Department of Allergy and Clinical Immunology, Cova da Beira University Hospital Centre, Covilhã, Portugal.

Address correspondence to Tiago Maricoto, Faculty of Health Sciences, University of Beira Interior, USF Aveiro-Aradas, Praceta Rainha D. Leonor, Aveiro 3800; Portugal. E-mail: tiago.maricoto@gmail.com

DOI: $10.1111 /$ jgs.15602 although effect on disease control and quality of life showed high discrepancy in the reported results, and all randomized studies revealed uncertainty in their risk of bias assessment.

CONCLUSION: All interventions seemed to improve inhaler performance and clinically relevant outcomes, but a placebo device could be the most effective. There is evidence that interventions reduce exacerbation risk in older adults, although to an overall moderate degree. J Am Geriatr Soc 00:1-10, 2018.

Key words: asthma; chronic obstructive pulmonary disease; inhalers

sthma and chronic obstructive pulmonary disease
(COPD) affect up to $10 \%$ of the population, and many
individuals with these conditions have uncontrolled symp-
toms. ${ }^{1}$ They experience frequent exacerbations, some of which
can be life threatening. Up to $53 \%$ of community-treated indi-
viduals may experience exacerbations, and good adherence to
therapy is associated with reductions in exacerbations in half
of cases. ${ }^{2-4}$ Inhaled therapy is the most widely used treatment,
but up to $90 \%$ of individuals used incorrect technique in clini-
cal studies, ${ }^{5}$ partly because the extensive variety of inhalers
and their technical specifications create significant barriers to
understanding of proper use. ${ }^{6}$ Although all available inhalers
may be equally efficient when properly used, ${ }^{7}$ there are vari-
ous device- and person-related factors that may significantly
influence performance. ${ }^{8-11}$ Poor inhaler technique is associated
with worse symptom control ${ }^{22,13}$ and leads to greater health-
care resource consumption and costs. ${ }^{14}$
Some studies have showed that teaching inhaler tech-
nique may lower the risk of exacerbations and death in these
individuals, ${ }^{2,3,15-17}$ but the effect of teaching decreases with
time, indicating the importance of regular reassessment.
There are many tools for teaching inhaler technique, ${ }^{20}$ and
two systematic reviews have addressed this. One concluded 
that there is lack of evidence about which is the best education method to improve inhaler technique. ${ }^{21}$ The other concluded that there is sufficient evidence of the efficacy of different inhaler educational strategies, but the authors did not quantify this effect with precision because it did not include a meta-analysis. ${ }^{22}$ In addition, neither review focused on older adults.

Inhaler technique performance is regarded as particularly complex in older adults with asthma or COPD, who also tend to have lower inhaler adherence rates. ${ }^{19,23,24}$ These individuals find it more difficult than younger adults to use the devices correctly, with several characteristics seeming to hamper them, such as cognitive impairment, low education level, osteoarthritis, and global frailty. ${ }^{23,25-28}$ For such reasons, and because these conditions are frequently underdiagnosed, elderly adults are more susceptible to disease consequences and exacerbations ${ }^{29,30}$ and are frequently excluded from clinical trials of education programs. Thus, there is a lack of evidence regarding the real effect that educational interventions have in these individuals. Our systematic review and meta-analysis assessed whether there is evidence that inhaler technique education in older adults with asthma or COPD improves clinical control and reduces disease exacerbations. We also analyzed which is the best method for teaching inhaler technique and how often it should be taught.

\section{METHODS}

\section{Eligibility Criteria}

Search Criteria Following a Population, Intervention, Comparator, Outcomes Format

\section{Participants}

We selected studies that included (not exclusively) participants aged 65 and older with asthma or COPD. In studies aggregating adults and older adults, we used the average age to decide on inclusion. Studies with mean age between 60 and 65 were included in preliminary analyses to assess their magnitude of influence on our major results and conclusions and were included in detailed analysis if considered highly relevant.

\section{Intervention}

We defined as the main criteria interventions that focus on teaching inhalation technique and, provided by health professionals and directed to patients or their caregivers, whatever method used (e.g., oral instructions and physical demonstration with placebo device, text-based print resources, media, educational tools (e.g., turbutest, InCheckDial), e-health interventions, combinations thereof). We included studies involving hospital staff (e.g., clinicians, nurses), pharmacists, general practitioners, community health workers, and others as providers.

\section{Comparator}

Different methods were compared with one another or placebo or usual care (treatment provided in a real-world scenario, according to local guidelines or healthcare provider judgments).

\section{Outcomes}

We included studies that addressed any of the following outcomes: inhaler performance evaluation (change from baseline scores preferred); all-cause hospitalization or allcause mortality; exacerbation rate or loss of control; clinical control (preferably measured on a validated scale); quality of life (preferably measured on a validated scale); functional control (e.g., as change from baseline scores in forced expiratory volume in the first second $\left(\mathrm{FEV}_{1}\right)$, forced vital capacity (FVC), $\mathrm{FEV}_{1} / \mathrm{FVC}$ ratio, peak expiratory flow $(\mathrm{PEF})$ )

\section{Types of studies}

We searched systematic reviews, meta-analyses, randomized controlled trials (RCTs), nonrandomized clinical trials, and quasi-experimental studies. We included quasi-randomized studies because of the lack of RCTs to reinforce the quality of our review and confidence in our findings.

\section{Search Methods}

We used the EMBASE, CENTRAL, and MEDLINE databases as primary sources and reference lists from studies included in primary sources and those found by author review and expert opinion as secondary sources. We also screened the main trial registry databases, such as the U.S. National Institutes of Health Ongoing Trials Register ClinicalTrials.gov (www.clinicaltrials.gov) and the World Health Organization International Clinical Trials Registry Platform (apps.who.int/trial search).

We used the Medical Subject Heading terms "nebulizers and vaporizers," "asthma," and "pulmonary disease, chronic obstructive," with a time limit for publication of March 2017.

Overall, we intended to reproduce the same search strategy of previous systematic reviews that addressed the same questions (see detailed search strategies in Supplementary Appendix S1).

\section{Selection Process}

Two independent, blinded authors (TM, LM) selected the articles according to the defined criteria and applied the following filter stages: cleaning of duplicated articles, selection of articles according to eligibility criteria and by reading the title and abstract, and selection of articles according to fulltext reading.

Reasons for article rejection are expressed in a Preferred Reporting Items for Systematic Reviews and MetaAnalyses (PRISMA) diagram ${ }^{31}$ (Figure 1). All disagreements, at every stage, including selection of studies, quality assessment, and data extraction, were resolved through discussion or by a third review author (LT-B).

\section{Data Collection Process}

Two authors (TM, LM) collected data from selected articles in their original presentation and noted them in a spreadsheet. We also collected indirect data from figures and 


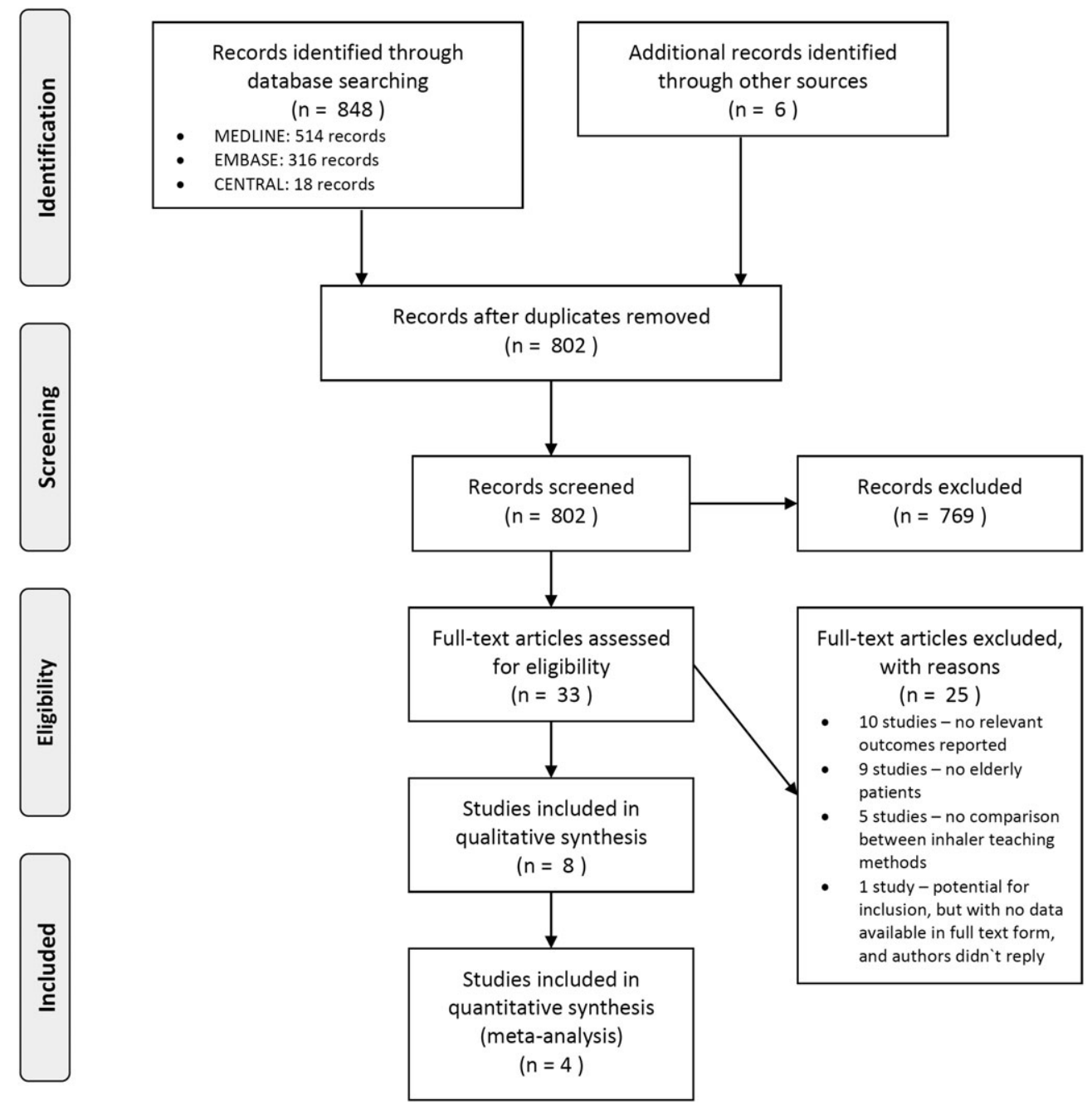

Figure 1. Flow diagram on search and article inclusion, according to Preferred Reporting Items for Systematic Reviews and MetaAnalysesstatement. ${ }^{31}$

charts, adapting their interpretation by consensus, and authors of original articles were contacted for further information and data.

\section{Type of Data Collected}

Two authors (TM, LM) collected the following information.

General data: Year, study type, number of participants, age, sex, follow-up time, withdrawals, diagnosis, disease severity, type of intervention, study location, time between interventions, type of intervention provider, adverse events and outcomes reported.

Outcomes: Inhaler performance, adherence rate, clinical control, quality of life (in any type of validated scale) and functional control $\left(\mathrm{FEV}_{1} \%\right.$; $\mathrm{FVC} \%, \mathrm{PEF} \%, \mathrm{FEV}_{1} / \mathrm{FVC}$ ratio) (in median, range, 95\% confidence interval (CI), standard deviation, standard error, or any index of percentage of change) and exacerbation rate, hospitalization, and mortality (in odds ratio, risk ratio (RR), hazard rate, number needed to treat, and their respective $95 \%$ CIs).

One author (TM) inserted data into software for preparing and maintaining Cochrane Reviews (Review Manager (RevMan), http://community.cochrane.org), and data were double-checked for correct entry.

\section{Analysis of Results and Assessment of the Risk of Bias}

Two authors (TM, LM) analyzed the data according to risk of bias using a qualitative approach. Quality of evidence for the collected outcomes of interest and recommendation for the interventions were assessed using the Grading of Recommendations, Assessment, Development and Evaluations (GRADE) system, as reported in the Cochrane Handbook for Systematic Reviews of Interventions. ${ }^{32}$ Two other authors (LT-B or JCS) confirmed this assessment.

Risk of bias was assessed in the following domains: random sequence generation, allocation concealment, blinding of participants and personnel, blinding of outcome assessment, incomplete outcome data, selective outcome reporting, and other biases.

Risk of bias in each study was graded as high, low, or uncertain and justifications for such judgment were reported in the "Risk of bias" table [Supplementary Appendix S2].

Publication bias was analyzed using a funnel plot.

\section{Measures of Treatment Effect}

Two authors (TM, JG) quantitatively analyzed RCTs to determine effect estimations, heterogeneity, and consistency 


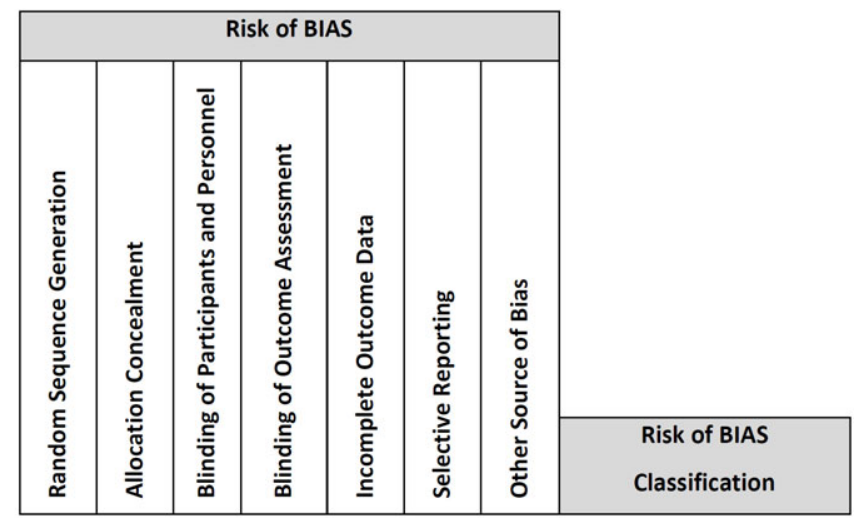

\begin{tabular}{|c|c|c|c|c|c|c|c|c|c|c|}
\hline \multirow{4}{*}{ 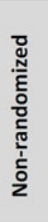 } & \multirow{4}{*}{ 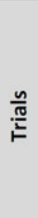 } & Mulhall 2016 & - & - & - & - & ? & $?$ & ? & High \\
\hline & & Lee 2016 & - & - & - & - & $?$ & ? & $?$ & High \\
\hline & & Takemura 2013 & - & - & - & - & + & + & ? & High \\
\hline & & Buist 2006 & - & - & - & - & $?$ & $?$ & $?$ & High \\
\hline \multirow{4}{*}{ 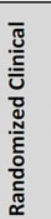 } & \multirow{4}{*}{$\frac{n}{\frac{\pi}{2}}$} & Tommelein 2014 & + & + & ? & ? & + & $?$ & $?$ & Uncertain \\
\hline & & Khdour 2009 & + & - & - & - & + & + & $?$ & High \\
\hline & & Rootmensen 2008 & + & + & $?$ & $?$ & + & - & $?$ & Uncertain \\
\hline & & Bourbeau 2003 & + & + & ? & ? & + & ? & ? & Uncertain \\
\hline
\end{tabular}

Figure 2. Risk of bias assessment in included studies according to Grading of Recommendations, Assessment, Development and Evaluations tool and recommended by Cochrane. ${ }^{32}$

tests. We used Mantel-Haenzsel RRs with 95\% CIs using a random-effects model for dichotomous data. Continuous outcomes were analyzed as standardized mean differences with 95\% CIs using a random-effects model, because the included studies used different measurement instruments. We used meta-analysis only for RCTs. Heterogeneity between effect sizes of included studies was assessed using visual inspection of forest plots and the chi-square test for heterogeneity (with $\mathrm{P}<.10$ ), and inconsistency between trials was described according to percentage of variability in effect estimates due to heterogeneity rather than chance $\left(\mathrm{I}^{2}\right)$. We also performed sensitivity analyses of the included studies and their effect on the meta-analysis. Results of the primary outcome, exacerbation risk reduction, with trial sequential analysis were also presented using the O'Brien Fleming monitoring boundaries approach. This was performed considering the results pooled in meta-analysis to exclude falsepositive or false-negative results from our review. ${ }^{33}$

No subgroup analysis was planned because of the small number of studies. Quantitative analyses were not performed in quasirandomized studies because of their high risk of bias.

All statistical procedures were performed using RevMan, GRADEPro online (https://gradepro.org/), and trial sequential analysis software provided by the Copenhagen Trial Unit (http://www.ctu.dk).

\section{RESULTS}

\section{Description of Studies}

Our search yielded 854 articles (Figure 1). From the 802 unique articles, eight studies met the inclusion criteria and were analyzed. Most studies were excluded because they did not address inhaler education in elderly adults or because no relevant outcomes were used. One study presented potential criteria for inclusion in its abstract, but no data from outcomes were available in full-text format, so it was excluded. ${ }^{34}$ One included study evaluated quality of life, but those results were not published in the article. ${ }^{35}$ Authors of these publications were contacted but did not reply.

Of the eight studies included, four had a randomized design, ${ }^{16,35-37}$ and the remaining four had a quasi-randomized pre- and post-intervention design ${ }^{17,38-40}$ (details in Supplementary Appendix S3).

1812 participants were evaluated. Five studies addressed only COPD, one addressed asthma, and two addressed both. Five studies were performed in secondary health care facilities, two at community pharmacies and one at primary health care centers. A pharmacist performed the educational intervention in half of the studies and a nurse or a doctor in the other half. Follow-up varied from 1 month to 2 years, and half of the studies had at least 1 year of follow-up. The mean age of participants was slightly greater than 65 in six of the studies and younger than 65 in the other two. We decided to include them to reinforce the quality of our review and confidence in our findings because the search strategy yielded few studies. Also, these two studies had large sample sizes ${ }^{39}$ and reported exacerbation risk as an outcome. ${ }^{35}$

Educational interventions varied between studies. Three studies examined a physical demonstration with placebo devices, which was the most frequent type of intervention, covering more than half of the total amount of participants. Two studies used video demonstration, and another two 


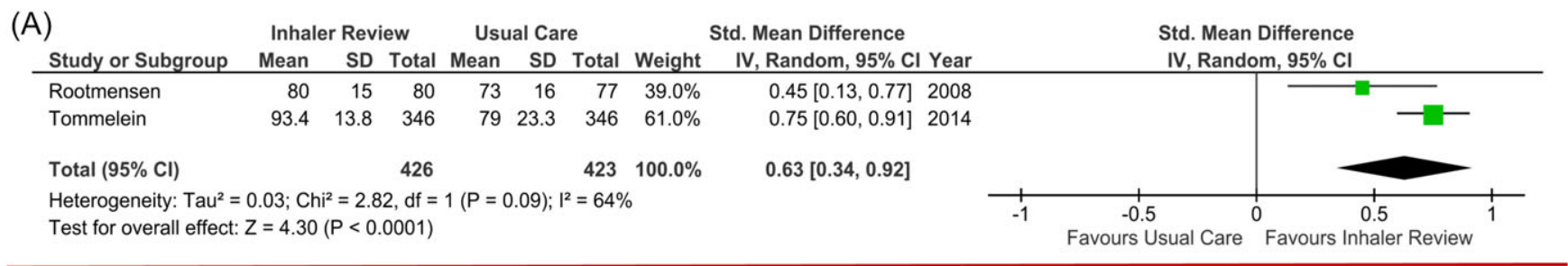

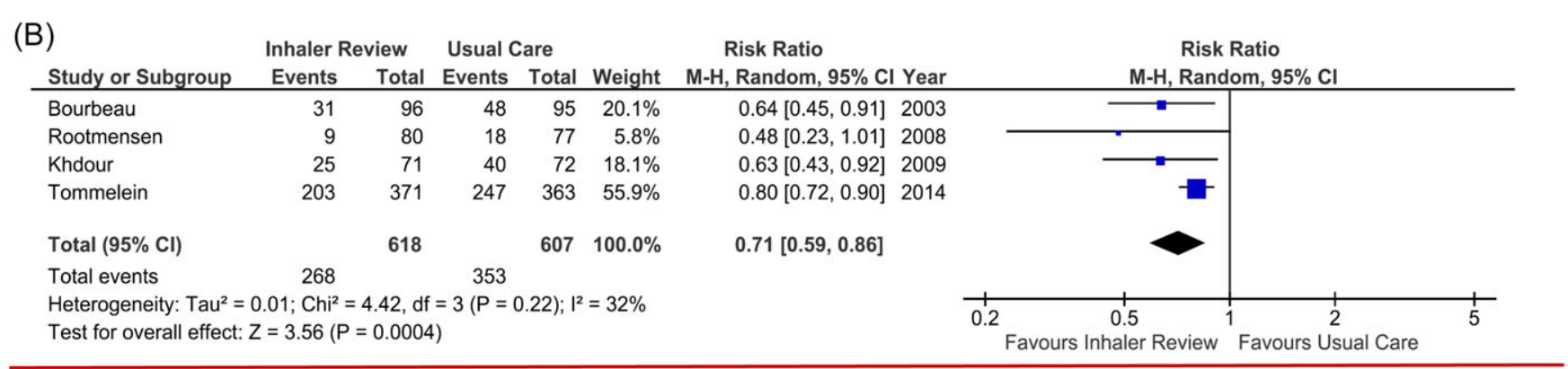

(C)

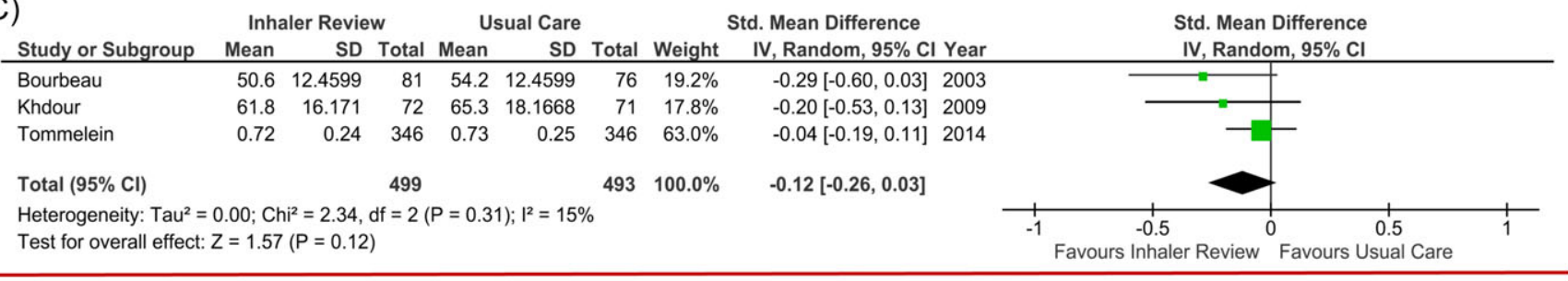

\section{(D)}

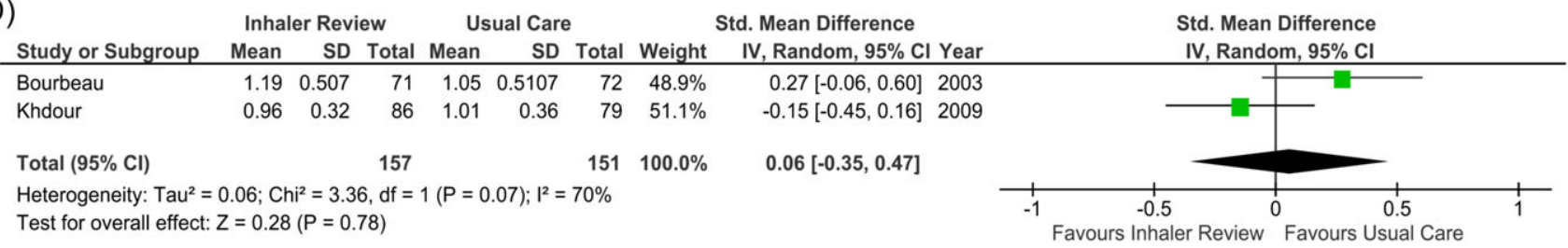

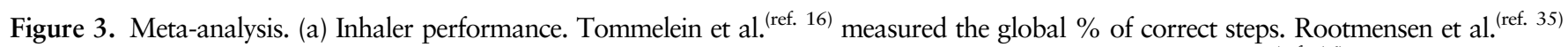
measured the global score of correct performance; (b) Exacerbation Rate. (c) Quality of Life. Tommelein et al. ${ }^{\text {(ref. } 16)}$ used ED-5D scale. Khdour et al. ${ }^{\text {(ref. } 36)}$ and Bourbeau et al. ${ }^{\text {(ref. } 37)}$ used St. George questionaire. (d) Respiratory function (change in forced expiratory volume in 1 second).

studies delivered written information. One study did not specify inhaler education type, ${ }^{35}$ and another one was unclear. ${ }^{17}$

Quality of life and exacerbations were the most commonly reported main outcomes (six studies), but different instruments and scales were used. ${ }^{16,17,35-37,40}$ Similar limitations occurred with clinical and functional control, adherence rate, and inhaler performance evaluation. Cost-effectiveness was never reported.

Most studies examined several aspects of intervention in addition to inhaler technique education: self-management plans, disease knowledge, and management of exacerbations and their triggers. Only two studies included a repeated education program, providing intervention every 6 months. ${ }^{17,40}$ All the other studies provided the intervention only at baseline.

\section{Risk of Bias in Included Studies}

Two independent reviewers (TM, LM) evaluated risk of bias of the included studies, reaching consensus in all evaluations (Figure 2). Nonrandomized trials were classified as being at high risk of bias in the main parameters, such as random sequence generation, allocation concealment, blinding of participants and personnel, and blinding of outcome assessment. In our review, RCTs showed an overal uncertainty in their risk assessment, although most of them had good blinding on random sequence generation and allocation concealment. The main limitation of RCTs was lack of blinding of the intervention and outcome assessment (detailed evaluation in Supplementary Appendix S2).

Logistic regression tests were used to determine whether there was a statistically significant relationship between lower risk of bias and magnitude of effect in the main reported outcomes. To build the model, we set all variables as binary: risk of bias ( $0=$ high, $1=$ uncertain $)$ and inhaler performance, adherence rate, symptom control, respiratory function, quality of life, and exacerbation rate ( 0 = negative outcome, $1=$ positive outcome). None of these outcome variables was statistically associated with risk of bias of included studies. 


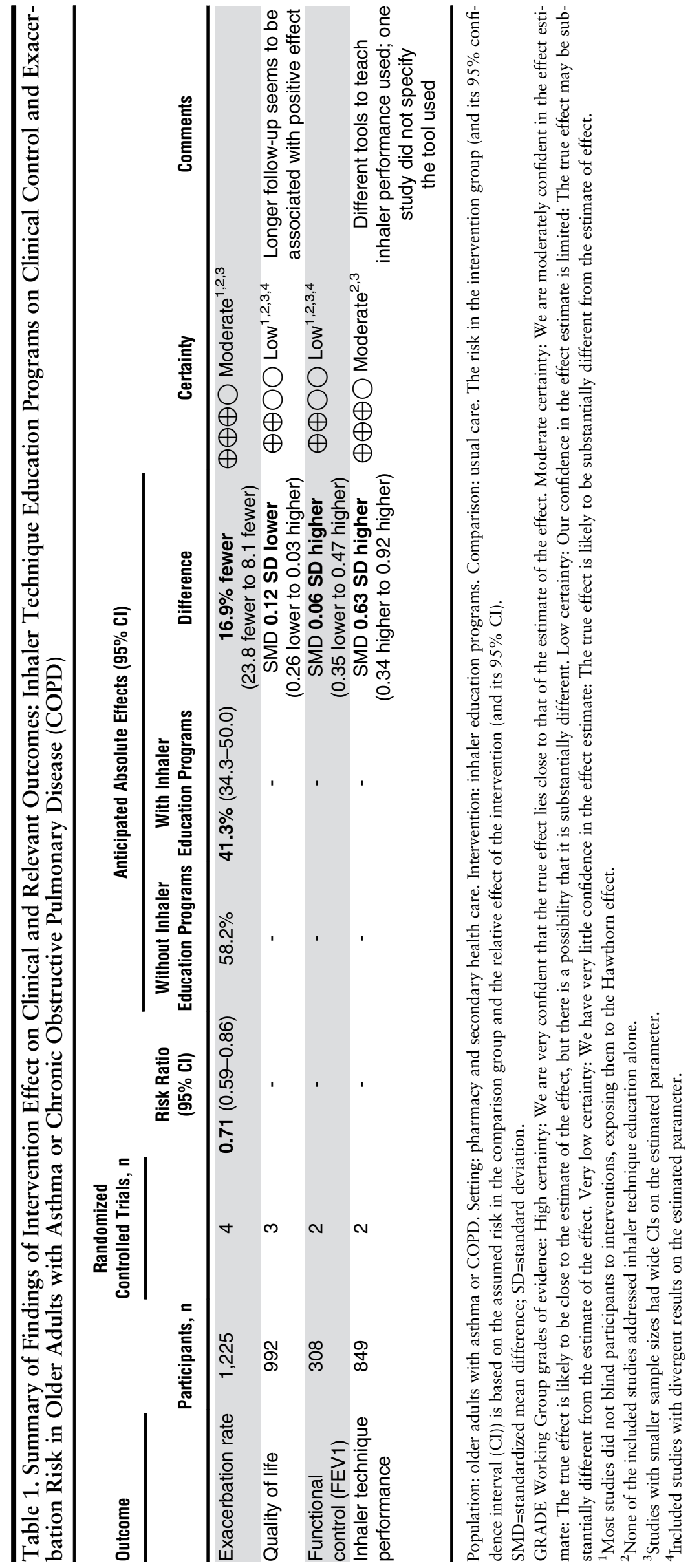




\section{Effects of Interventions}

Table 1 shows the main findings of clinical and relevant outcomes from selected studies, and Figure 3 shows the results of the meta-analysis.

\section{Inhaler Performance}

Only half of the studies measured the effect of the intervention on inhaler performance, and all ${ }^{16,38,40}$ except one ${ }^{35}$ showed improvement. That one study did not specify the type of tool used to teach inhaler technique, and it may not have been the primary objective of the intervention. Authors were contacted but did not reply. Although significant differences were not found after the intervention, when results were pooled in a meta-analysis together with a $\mathrm{RCT}^{16}{ }^{16}$ they showed a significant effect on inhaler performance. Nevertheless, heterogeneity was high, probably because different measures of inhaler performance were used. Also, the one study ${ }^{35}$ found a significant reduction in exacerbation rate at the end of follow up, which could be due to the additional topics covered in the intervention, especially disease self-management skills.

\section{Exacerbation Rate}

All RCTs resulted in a significant reduction in exacerbation rates, in the intervention group, and comparing with usual care group. ${ }^{16,35-37}$ The reported mean RR on these studies varied from 0.45 to 0.82 , with wide CIs and favouring the intervention group. Pooling these results in a meta-analysis, we found a significant mean reduction of almost $30 \%$ in exacerbation rates favouring the intervention. ( $R R=0.71$, $95 \% \mathrm{CI}=0.59-0.86)$ and a significant low heterogeneity index. In addition, sensitivity analysis showed that removing any of the included studies did not affect the outcome. Also, trial sequential analysis (Supplementary Appendix S4) confirmed our confidence in these findings, excluding the risk of a false-positive result, because significant boundaries and the necessary sample size were achieved.

One quasirandomized study had the same results, with relative reduction in exacerbation rates of almost $50 \% .{ }^{17}$ One study did not show any differences. ${ }^{40}$ This was a randomized study, comparing the use of a peak flow meter with symptom monitoring as a basis for disease control, and used physical demonstration with placebo devices in all participants, repeated every 6 months and provided by a clinician. It had a large sample size (396 participants) and 24 months of follow-up and participants had predominantly moderate to severe asthma. The authors did not find a reduction in global exacerbation rate, which could be because this study included only individuals with asthma, whereas participants in the other studies had COPD or both.

\section{Disease Control}

All studies evaluated the effect of intervention on several aspects of disease control, mainly on quality of life and symptoms. A although the results were highly discrepant because half of the studies found improvement in these outcomes $^{36,37,39,40}$, whereas the other half did not ${ }^{16,17,35,38}$. Meta-analysis including only RCTs did not find significant improvement in quality of life, although a sensitivity analysis including only the two studies that used the St. George's
Respiratory Questionnaire ${ }^{36,38}$ showed an improvement of 3.57 points in mean score $(95 \% \mathrm{CI}=0.36-6.78)$. No relevant characteristics seemed to differentiate the studies with regard to these findings, but detailed analysis of the RCTs showed that longer follow-up was associated with a more significant positive effect on quality of life. ${ }^{36,38}$ Nevertheless, the magnitude of these effects was small, and with wide CIs, and was associated with overall moderate strength of evidence.

\section{Respiratory Function}

Half of the studies evaluated effect on $\mathrm{FEV}_{1}$, but only one showed a significant benefit of the intervention. ${ }^{40}$ In this study, inhaler education was not the primary objective, and all participants received a thorough disease self-management program, with several intervention aspects in addition to inhaler education. The magnitude of the observed effect was small, with a wide CI. Quantitative analysis did not show any significant benefit either.

\section{Adherence}

Three studies evaluated the effect on adherence to inhaled medication, and all showed significant improvement after the intervention. ${ }^{16,17,36}$ This effect may also be due to the Hawthorne effect. Only two of the RCTs evaluated this outcome, but they used different scales, and one did not report useful results for quantitative analysis.

\section{Education Frequency}

Only two studies included a repeated education program, which was mainly on a biannual basis. ${ }^{1740}$ Both were nonrandomized and reported divergent results in the main outcomes. Thus, we could not perform a quantitative or sensitivity analysis on them, which limits any kind of conclusion about how often inhaler review and education should be recommended.

\section{DISCUSSION}

\section{Summary of Main Results}

The main finding of our review is that inhaler technique education can significantly reduce exacerbation risk; this is reinforced by a significant pooled result with a low index of statistical heterogeneity in the meta-analysis. This is the first study to find such results in older adults with asthma or COPD.

Interventions can also improve quality of life and clinical control, but results are still divergent. Also, by enhancing self-management education, adherence also increases, but this was difficult to quantify. These findings should be interpreted with caution because most studies lack sufficient quality of the evidence on their results, and this is due to several limitations in design and methods that introduce a high risk of bias.

Most studies addressed complex intervention aspects beyond inhaler technique education alone, and this is particularly relevant to the outcomes of interest because it makes it harder to determine the true effect of an inhaler education approach alone. Thus, this review failed to uncover any important information about the role of inhaler technique education alone. 
Although many guidelines recommend regular inhaler review, it is unclear how often that should be performed with older adults.

Only one RCT included individuals with asthma, ${ }^{35}$ and the others only had participants with COPD. Thus, individuals with asthma accounted for only $6 \%$ of total analyzed individuals, which skews the available evidence toward COPD.

We could not perform additional subgroup analysis, namely according to age strata (such as in patients below or above 75 years old), or even according to important comorbidities (e.g., osteoarthritis, frailty, cognitive disorders). All studies had a mean age of younger than 70 and none reported such data. Such subgroup analysis would be clinically relevant because there is increasing evidence suggesting that such characteristics seem to be determinant to inhaler performance and to disease outcomes in these individuals. ${ }^{23,25,27,28}$

\section{Overall Applicability and Quality of Evidence}

Using the GRADE approach to rate the quality of the evidence, our analysis showed a significant overall risk of bias in studies. Half did not have a randomized design, and even RCTs were not blinded to the intervention because of its intrinsic nature. This introduces a potential Hawthorne effect, which could overestimate the main outcomes. Although this could compromise the internal validity of trials, globally, all showed a regular and similar trend in the results, indicating their external validity and applicability.

Although the included studies did not perform any cost-effective analysis or report adverse effects of the interventions, the potential benefits may outweigh the risks, which also favors regular inhaler education. Several studies have highlighted these aspects. ${ }^{2,14,15}$

It is difficult, with the existing evidence, to determine the true potential of inhaler education alone or what the most efficient education method is. Some studies suggested that placebo device demonstration may be the best, ${ }^{4-43}$ but this was not clear in our review. It is possible that older adults have some resistance to this method because of problems such as cognitive impairment. ${ }^{23,26}$ We found only one RCT that used placebo device training for the intervention, and it showed improvement in exacerbation risk only for severe episodes. ${ }^{16}$ More randomized and blinded studies are needed to test different types of interventions, clarify which factors may influence inhaler performance, and assess the effect of performance on clinically relevant outcomes in older adults.

Well-trained staff with adequate time dedicated to instruction performed these studies. This does not usually happen in the real world, where health professionals are on tight schedules and do not have proper training on handling all available devices. This could undermine generalizability of study findings.

\section{Potential Biases in the Review Process}

Our review process was based on Cochrane recommendations $^{32}$ and is in accordance with the PRISMA statement, ${ }^{31}$ which makes it less susceptible to major biases and errors. The search method was based on main databases (CENTRAL, MEDLINE, EMBASE) and covered important secondary sources. Criteria used were broad, yielding a representative selection of studies. Previous systematic reviews $^{21,22}$ on the same topic were also screened for secondary sources of important studies, which minimizes the risk of bias in this process. During the selection process, in addition to RCTs, we included nonrandomized studies to avoid underestimation of true effects of inhaler education programs. Although the quality of evidence from nonrandomized studies is poor, we believe that all four studies included helped reinforce the strength of recommendation regarding some of the outcomes, namely exacerbation risk reductions. In addition, by including quasirandomized studies, we highlight the need for further, adequately designed research in this population.

Two independent reviewers assessed quality of evidence using the GRADE approach, and agreement was obtained in all studies. Two other authors confirmed the process. In addition, the main results of this analysis are similar to those reported in previous systematic reviews. ${ }^{21,22}$

Performing a meta-analysis of such different and complex interventions could lead to false interpretation of results, because these studies are not truly comparable. To overcome this, we confirmed the main meta-analysis findings using trial sequential analysis (Supplementary Appendix S4), which strongly reinforced the finding of a significant reduction of exacerbations, increasing confidence in the results and excluding risk of a false-positive result.

\section{Comparison with Other Studies and Reviews}

To our knowledge, this is the first systematic review of inhaler education in older adults with asthma or COPD that obtained such clinically relevant results on disease control, namely the reduction of exacerbation risk. Given the aging of the population and the complex characteristics of these individuals, we find this work relevant and timely.

This systematic review stands out from previous ones for several reasons. ${ }^{21,22}$ First, it focuses on older adults, who often have poor clinical control, are more susceptible to exacerbations, and often have poor inhaler technique. ${ }^{25,26}$ In addition, we included randomized and nonrandomized studies, which allowed us to make realistic conclusions about the effect of inhaler technique education. Also, our review included recent studies that were not included in previous work. Finally, our systematic review highlights that most studies showed a positive effect of training on exacerbation risk reduction.

In a systematic review, ${ }^{21}$ the authors included only individuals with asthma and included adults and older adults together, without performing a subgroup analysis of the latter. This spread of age ranges may have introduced some bias, mainly derived from endotypic and phenotypic disease differences, which could lead to different clinical responses to education on inhaler performance. Another systematic review ${ }^{22}$ focused on postintervention inhaler performance as the main outcome but did not fully analyze the clinically relevant outcomes. Also, it included a wide age range, did not perform a subgroup analysis of older adults, and did not include a meta-analysis. In any case, previous work and our review indicate that inhaler technique education improves performance and has a potential benefit for clinical outcomes, although the strength of the evidence is low to moderate. 
We found inconsistent results in clinical control and quality of life, but overall, interventions seem to significantly reduce exacerbation rates. This is particularly relevant for health economics, and several studies have shown positive, cost-effective associations in this field..$^{2,14,15}$

\section{CONCLUSIONS}

Inhaler technique education is a critical aspect of selfmanagement programs, and different types of interventions seem to improve inhaler performance and clinically relevant outcomes. Review of inhaler technique is recommended, and there is evidence that interventions that promote improvement in inhaler technique ameliorate disease control. In addition, these interventions significantly reduce exacerbation risk in older adults, although the strength of evidence for these outcomes is moderate. Further studies are warranted to compare education methods and target populations and to define the best regular follow-up.

\section{DIFFERENCES BETWEEN PROTOCOL AND REVIEW}

The protocol of this systematic review was registered in PROSPERO with the number CRD42017063847, available at: https://www.crd.york.ac.uk/PROSPERO/display_record. asp?ID=CRD42017063847.

The study protocol differs from the final work only in the age criteria. In the protocol, we intended to include only studies with a mean age of participants aged 65 and older but included two studies with a mean age slightly below that because we found them very relevant to our objective, and both reported important outcomes.

\section{ACKNOWLEDGMENTS}

The authors than Prof. Jonh Groarke, Harvard Medical School, for his scientific support and input in reviewing the final version of this manuscript.

Conflict of Interest: The authors declare no conflict of interests.

Author Contributions: Study design: TM, LT-B. Data extraction: TM, LM. Data review and analysis: all authors. Statistical tests in first approach: TM, JG. First draft: TM, LM. Comments on first draft: all authors. LT-B and JCS are the guarantors of the study.

Financial Disclosure: The academic affiliation of this systematic review is the Life and Health Sciences Research Institute/3B's, University of Minho and the Faculty of Health Sciences, University of Beira Interior. This work was prepared with scientific support from Harvard Medical School, in the context of the Portuguese Clinical Scholars Research Training Program.

Sponsor's Role: None.

\section{REFERENCES}

1. World Health Organization. The Global Burden of Disease: 2004 Update (online). Available at http://www.who.int. [Accessed: November 2017]

2. van Boven JF, Tommelein E, Boussery $\mathrm{K}$, et al. Improving inhaler adherence in patients with chronic obstructive pulmonary disease: A cost-effectiveness analysis. Respir Res 2014;15:66.
3. Vestbo J, Anderson JA, Calverley PM, et al. Adherence to inhaled therapy, mortality and hospital admission in COPD. Thorax 2009;64:939-43.

4. Engelkes M, Janssens HM, de Jongste JC, Sturkenboom MCJM, Verhamme KMC. Medication adherence and the risk of severe asthma exacerbations: A systematic review. Eur Respir J 2015;45:396-407.

5. Lavorini F, Magnan A, Dubus JC, et al. Effect of incorrect use of dry powder inhalers on management of patients with asthma and COPD. Respir Med 2008;102:593-604.

6. Haughney J, Price D, Barnes NC, Virchow JC, Roche N, Chrystyn H. Choosing inhaler devices for people with asthma: Current knowledge and outstanding research needs. Respir Med 2010;104:1237-1245.

7. Dolovich MB, Ahrens RC, Hess DR, et al., American College of Chest Physicians., American College of Asthma, Allergy, and Immunology. Device selection and outcomes of aerosol therapy: Evidence-based guidelines: American College of Chest Physicians/American College of Asthma, Allergy, and Immunology. Chest 2005;127:335-371.

8. Broeders ME, Molema J, Hop WC, et al. Inhalation profiles in asthmatics and COPD patients: Reproducibility and effect of instruction. J Aeros Med 2003;16:131-141.

9. Crompton GK, Barnes PJ, Broeders M, et al., Aerosol Drug Management Improvement Team. The need to improve inhalation technique in Europe: A report from the Aerosol Drug Management Improvement Team. Respir Med 2006;100:1479-1494.

10. Maricoto T, Rodrigues LV, Teixeira G, Valente C, Andrade L, Saraiva A. Assessment of inhalation technique in clinical and functional control of asthma and chronic obstructive pulmonary disease. Acta Med Port 2015;28: 702-707

11. Rootmensen GN, van Keimpema AR, Jansen HM, et al. Predictors of incorrect inhalation technique in patients with asthma or COPD: A study using a validated videotaped scoring method. J Aerosol Med Pulm Drug Deliv 2010; 23:323-328.

12. Melani AS, Bonavia M, Cilenti V, et al., Gruppo Educazionale Associazione Italiana Pneumologi Ospedalieri. Inhaler mishandling remains common in real life and is associated with reduced disease control. Respir Med 2011; 105:930-938.

13. Westerik JA, Carter V, Chrystyn H, et al. Characteristics of patients making serious inhaler errors with a dry powder inhaler and association with asthma-related events in a primary care setting. J Asthma 2016;53:321-329.

14. Roggeri A, Micheletto C, Roggeri DP. Inhalation errors due to device switch in patients with chronic obstructive pulmonary disease and asthma: Critical health and economic issues. Int J Chron Obstruct Pulmon Dis 2016;11: 597-602.

15. Simoni-Wastila L, Wei YJ, Qian J, et al. Association of chronic obstructive pulmonary disease maintenance medication adherence with all-cause hospitalization and spending in a Medicare population. Am J Geriatr Pharmacother 2012;10:201-10.

16. Tommelein E, Mehuys E, Van Hees T, et al. Effectiveness of pharmaceutical care for patients with chronic obstructive pulmonary disease (PHARMACOP): A randomized controlled trial. Br J Clin Pharmacol 2014;77:756-766.

17. Takemura M, Mitsui K, Ido M, et al. Effect of a network system for providing proper inhalation technique by community pharmacists on clinical outcomes in COPD patients. Int J Chron Obstruct Pulmon Dis 2013;8: 239-244

18. Broeders ME, Sanchis J, Levy ML, Crompton GK, Dekhuijzen PN, ADMIT Working Group. The ADMIT series-Issues in inhalation therapy. 2. Improving technique and clinical effectiveness. Prim Care Resp J 2009;18:76-82.

19. van der Palen J, Klein JJ, Kerkhoff $\mathrm{AH}$, et al. Inhalation technique of 166 adult asthmatics prior to and following a self-management program. J Asthma 1999;36:441-447.

20. Price D, Bosnic-Anticevich S, Briggs A, et al. Inhaler competence in asthma: Common errors, barriers to use and recommended solutions. Respir Med 2013;107:37-46.

21. Normansell R, Kew KM, Mathioudakis AG. Interventions to improve inhaler technique for people with asthma. Cochrane Database Syst Rev 2017;3:CD012286.

22. Klijn SL, Hiligsmann M, Evers S, et al. Effectiveness and success factors of educational inhaler technique interventions in asthma \& COPD patients: A systematic review. NPJ Prim Care Resp Med 2017;27:24.

23. Allen SC, Jain M, Ragab S, Malik N. Acquisition and short-term retention of inhaler techniques require intact executive function in elderly subjects. Age Ageing 2003;32:299-302.

24. Barrons R, Pegram A, Borries A. Inhaler device selection: Special considerations in elderly patients with chronic obstructive pulmonary disease. Am J Health Syst Pharm 2011;68:1221-1232.

25. Quinet P, Young CA, Heritier F. The use of dry powder inhaler devices by elderly patients suffering from chronic obstructive pulmonary disease. Ann Phys Rehabil Med 2010;53:69-76. 
26. Allen SC, Warwick-Sanders M, Baxter M. A comparison of four tests of cognition as predictors of inability to learn to use a metered dose inhaler in old age. Int J Clin Pract 2009;63:1150-1153.

27. Aydemir Y. Assessment of the factors affecting the failure to use inhaler devices before and after training. Respir Med 2015;109:451-458.

28. Gray SL, Williams DM, Pulliam CC, Sirgo MA, Bishop AL, Donohue JF. Characteristics predicting incorrect metered-dose inhaler technique in older subjects. Arch Intern Med 1996;156:984-988

29. Enright PL, McClelland RL, Newman AB, et al. Underdiagnosis and undertreatment of asthma in the elderly. Cardiovascular Health Study Research Group. Chest 1999;116:603-613

30. Smith AM, Villareal M, Bernstein DI, Swikert DJ. Asthma in the elderly: Risk factors and impact on physical function. Ann Allergy Asthma Immunol 2012;108:305-310.

31. Moher D, Liberati A, Tetzlaff J, Altman DG, The PRISMA Group. Preferred reporting items for systematic reviews and meta-analyses: The PRISMA statement. PLoS Med 2009;6:e1000097.

32. Higgins JP, Green S, eds. Cochrane Handbook for Systematic Reviews of Interventions Version 5.1. : The Cochrane Collaboration; 2011. Available at https://training.cochrane.org.

33. Wetterslev J, Thorlund K, Brok J, Gluud C Trial sequential analysis may establish when firm evidence is reached in cumulative meta-analysis. J Clin Epidemiol 2008;61:64-75.

34. Kishan J, Garg K. Effect of health education on compliance in asthma. J Indian Med Assoc 2012;110:700, 702-705

35. Rootmensen GN, van Keimpema AR, Looysen EE, et al. The effects of additional care by a pulmonary nurse for asthma and COPD patients at a respiratory outpatient clinic: Results from a double blind, randomized clinical trial. Patient Educ Couns 2008;70:179-186.

36. Khdour MR, Kidney JC, Smyth BM, McElnay JC. Clinical pharmacy-led disease and medicine management programme for patients with COPD. Br J Clin Pharmacol 2009;68:588-598.

37. Bourbeau J, Julien M, Maltais F, et al., Chronic Obstructive Pulmonary Disease axis of the Respiratory Network Fonds de la Recherche en Santé du Québec. Reduction of hospital utilization in patients with chronic obstructive pulmonary disease: A disease-specific self-management intervention. Arch Intern Med 2003;163:585-591.

38. Mulhall AM, Zafar MA, Record S, Channell H, Panos RJ A tablet-based multimedia education tool improves provider and subject knowledge of inhaler use techniques. Respir Care 2017;62:163-171.
39. Lee JY, Yoo KH, Kim DK, et al. Effects of educational interventions for chronic airway disease on primary care. J Korean Med Sci 2016;31: 1069-1074.

40. Buist AS, Vollmer WM, Wilson SR, Frazier EA, Hayward AD. A randomized clinical trial of peak flow versus symptom monitoring in older adults with asthma. Am J Respir Crit Care Med 2006;174:1077-1087.

41. Bosnic-Anticevich SZ, Sinha H, So S, et al. Metered-dose inhaler technique: The effect of two educational interventions delivered in community pharmacy over time. J Asthma 2010;47:251-256.

42. Crane MA, Jenkins CR, Goeman DP, Douglass JA. Inhaler device technique can be improved in older adults through tailored education: Findings from a randomised controlled trial. NPJ Prim Care Resp Med 2014; 24:14034.

43. Lavorini F, Levy ML, Corrigan C, Crompton G, ADMIT Working Group. The ADMIT series-issues in inhalation therapy. (6) Training tools for inhalation devices. Prim Care Resp J 2010;19:335-341.

\section{SUPPORTING INFORMATION}

Additional Supporting Information may be found in the online version of this article.

Appendix S1: Search Strategy Used

Appendix S2: Quality assessment and Risk of bias table.

Appendix S3: Complete data of selected studies.

Appendix S4: Trial sequential analysis on the primary outcome, exacerbation risk reduction, with a two-sided graph. The required information size to demonstrate or reject a $25 \%$ relative risk reduction in benefit on inhaler technique review with a usual care group proportion of $58.2 \%$, an alpha of $5 \%$ and a beta of $80 \%$ is 527 patients (vertical etched line), and according to heterogeneity index found in meta-analysis. The curved etched lines represent the trial sequential monitoring boundaries and the futility boundaries. The solid curved line is the cumulative $\mathrm{Z}$-curve. 